\title{
Gout: new insights into an old disease
}

\author{
Fabio Martinon ${ }^{1}$ and Laurie H. Glimcher,1,2
}

1Department of Immunology and Infectious Diseases, Harvard School of Public Health, and

2Department of Medicine, Harvard Medical School, Boston, Massachusetts, USA.

\begin{abstract}
Gout is an autoinflammatory disorder associated with deposition of monosodium urate (MSU) crystals in joints and periarticular tissues. Recent advances suggest that the innate immune system may drive the gouty inflammatory response to MSU. These findings prompt questions concerning how the innate immune system recognizes MSU and the identities of the receptors involved. In this issue of the JCI, Chen et al. show that the IL-1 receptor and its signaling protein myeloid differentiation primary response protein 88 (MyD88) but not the "classical" innate immune receptors, TLRs, are central for MSU-induced inflammation (see the related article beginning on page 2262).
\end{abstract}

\section{MSU in gout: a role for the innate immune system?}

Gout is considered one of the most painful acute conditions that human beings can experience and was among the first diseases recognized as a distinct entity. Through the ages, gout has captured the attention of many famous physicians, including the Egyptian Imhotep, who lived in the 27 th century BCE, and the revered Hippocrates (ca. 460-377 BCE), who made the distinction between gout (Greek: podagra) and other forms of arthritis (1). Gout is generally associated with hyperuricemia and is characterized by deposition of monosodium urate (MSU) crystals within the joints (2). Back in the 1960s, Faires and MacCarty, in a heroic effort to study the role of MSU crystals in gout, injected their left knee joints with $20 \mathrm{mg}$ of MSU crystals. Both rapidly developed an acute inflammation displaying all the characteristics of violent gouty attacks, that left them, 4 hours after injection, prostrate with excruciating pain. After recovering, they reported: "At this point we were impressed by the accuracy of Sydenham's [1683] classic description of gout" (3). Their experiment clearly demonstrated that MSU crystals are the initial trigger of gout and prompted the

Nonstandard abbreviations used: IL-1R, IL-1 receptor; MSU, monosodium urate; MyD88, myeloid differentiation primary response protein 88 ; NALP, NACHT, LRR, and pyrin domain-containing protein; NLR, NOD-like receptor; TIR domain, Toll/IL-1R domain; TIRAP, TIR domain-containing adaptor protein; TRAM, TRIF-related adaptor molecule; TRIF, TIR domain-containing adaptor-inducing IFN- $\beta$.

Conflict of interest: The authors have declared that no conflict of interest exists.

Citation for this article: J. Clin. Invest. 116:2073-2075 (2006). doi:10.1172/JCI29404. fundamental question as to how MSU crystals activate the immune system to drive inflammation. A recent breakthrough by Kenneth Rock's laboratory demonstrated that uric acid released from "damaged cells" forms MSU crystals, which act as a "danger signal” capable of maturing dendritic cells, driving antigen presentation and stimulating $\mathrm{T}$ lymphocytes (4). This finding was very exciting for two main reasons. First, it suggested a function for MSU crystals, beyond their role in gout, in facilitating an efficient immune response, as might occur in the course of tissue damage associated with infection (5). Second, Rock's observation revealed that the type of dendritic cell response elicited by MSU crystals strongly resembled the response elicited by exogenous adjuvants, a class of molecules that stimulate the innate immune system to promote antigen presentation and stimulate $T$ cell responses (6). These observations were therefore consistent with the hypothesis that MSU crystals directly activate the innate immune system.

\section{Role of the TLRs}

In this issue of the JCI, a new study from Rock's laboratory, by Chen et al., investigates the role of various components of the innate immune system in MSU-mediated inflammation (7). TLRs are the best characterized family of innate immune receptors. These transmembrane proteins sense structural components shared by many microbes and are particularly adept in activating dendritic cells, a crucial step that links the innate and adaptive immune responses (8). To investigate the role of TLRs, Chen et al. injected MSU crystals into the peritoneal cavity of either wild- type mice or mice deficient in various TLRs and monitored inflammation by quantifying neutrophil influx. Strikingly, none of the 9 TLR-deficient mouse strains analyzed showed an impairment in neutrophil influx, suggesting that TLRs may not be crucial for MSU-induced inflammation. To confirm these findings and rule out a possible role for TLR5 or TLR8 (since mice deficient in these TLRs are not available), the authors reconstituted a cell line with all the TLRs and monitored the ability of MSU crystals to activate these receptors. None of the 11 TLRs tested responded per se to stimulation by MSU crystals (7). Although these negative results do not rule out the possibility that an unidentified coreceptor may cooperate with a TLR to recognize MSU, taken together with the results of the knockout mice studies, they render the hypothesis that TLRs sense MSU unlikely.

\section{IL-1 $\beta$ and the IL-1 receptor: key players in MSU crystal- mediated inflammation}

A key structural motif involved in TLR signaling is the Toll/IL-1 receptor (TIR) domain. TIR can be found in the cytoplasmic portion of all TLRs, members of the IL-1 receptor (IL-1R) family, and a group of adaptor proteins including myeloid differentiation primary response protein 88 (MyD88), TIR domain-containing adaptor protein (TIRAP), TIR domain-containing adaptor-inducing IFN- $\beta$ (TRIF), and TRIF-related adaptor molecule (TRAM), all of which play fundamental roles in TLR and IL-1R signaling (9). When Chen et al. analyzed mice deficient in these adaptors, MSU crystal-induced inflammation was not impaired in TIRAP-, TRIF-, and TRAM-deficient mice but, surprisingly, was almost completely abolished by MyD88 deficiency (7). This prompted the authors to investigate the role of the IL-1Rs, the other receptor family that signals via MyD88, and led to the provocative observation that MSU crystal-induced inflammation requires IL-1R. This result was unexpected, considering that IL-1R is generally not believed to be an innate immune receptor, but makes sense in light 


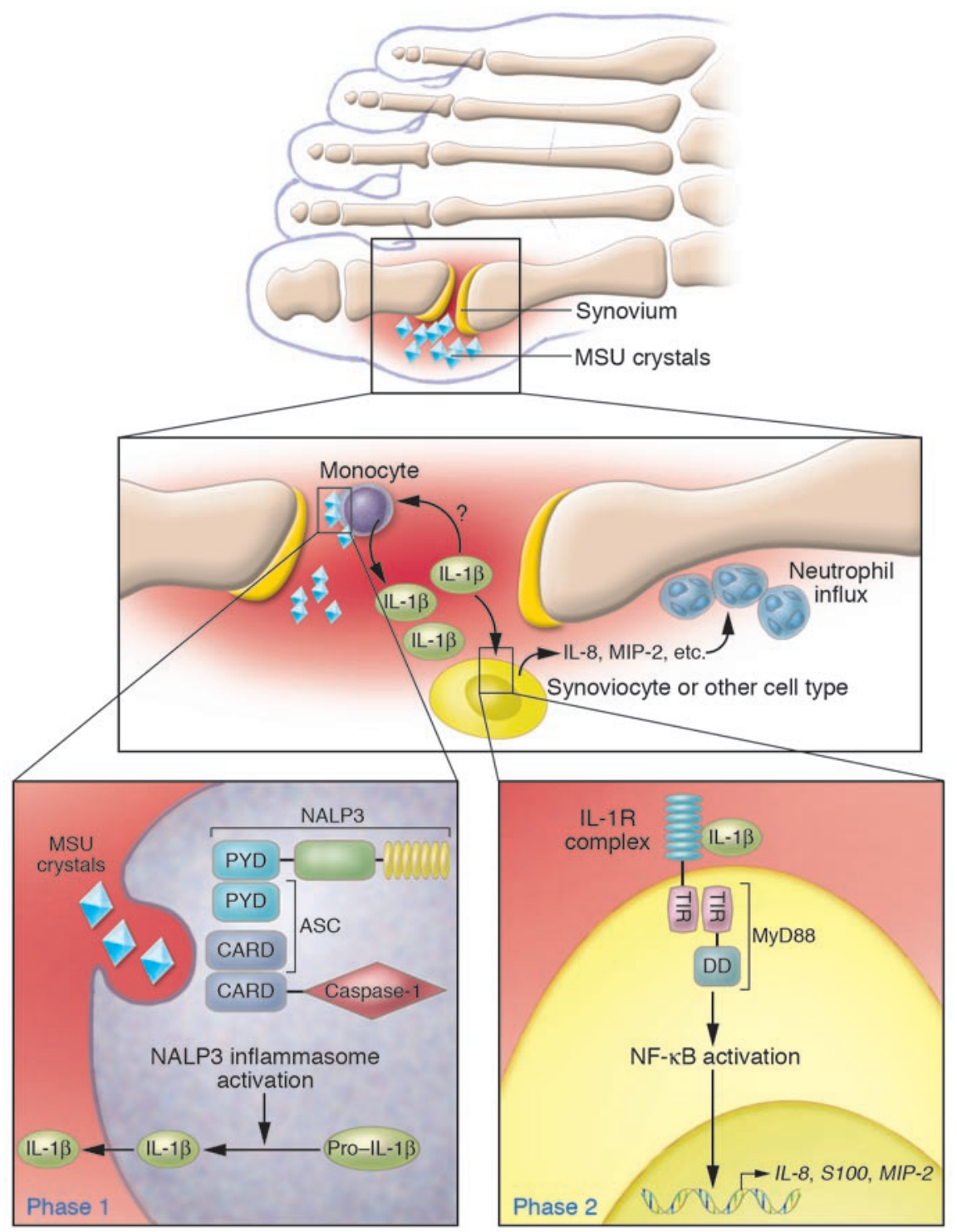

of recent insights into the mechanism that controls the activation of the IL-1R ligand IL- $1 \beta$. IL- $1 \beta$ is a proinflammatory cytokine whose production and activation involve 3 main steps: first, the production of the precursor pro-IL- $1 \beta$; second, the maturation of the precursor; and finally the secretion of the mature IL- $1 \beta$ into the extracellular space. The maturation is a very specific step that requires the activation of a protease, caspase-1, by a member of the NOD-like receptor (NLR) family. NLRs are intracellular receptors that detect microbes and constitute an emerging branch of the innate immune system (10). Some NLRs, such as NACHT, LRR, and pyrin domain-containing protein (NALP), neuronal apoptosis inhibitory protein (NAIP), and ICE protease-activat- ing factor (IPAF), form complexes called inflammasomes that are involved in the activation of caspase-1. Interestingly, MSU crystals were recently shown to activate NALP3, and macrophages deficient in various components of the NALP3 inflammasome are unable to secrete active IL- $1 \beta$ following MSU crystal stimulation (11). This finding is consistent with the concept that IL- $1 \beta$ induced by MSU crystals may then trigger inflammation through an IL-1R/MyD88-dependent pathway. Furthermore, using bone marrow chimera experiments, Chen et al. show that MSU crystal-mediated inflammation does not require IL-1R and MyD88 in bone marrow cells, suggesting that other cell types respond to IL-1 $\beta$ to transmit the inflammatory signal (7).

\section{Figure 1}

Model of the role of IL-1 $\beta$ in gouty inflammation. MSU crystals internalized by monocytes activate the NALP3 inflammasome (Phase 1, lower left). NALP3 protein activation leads to the recruitment and activation of the adaptor ASC and caspase-1 via PYD-PYD and CARD-CARD homotypic interactions, resulting in the processing and maturation of proIL-1 $\beta$ into its biologically active form, IL-1 $\beta$. IL-1 $\beta$ (mainly acting on nonleukocytic cell types, possibly synoviocytes) will then activate the IL-1R complex, leading to recruitment of MyD88 via TIR-TIR homotypic interactions. This results in the activation of NF-KB, which will turn on the transcription of neutrophilrecruiting chemokines, such as IL-8, S100, or macrophage inflammatory protein 2 (MIP-2) (Phase 2, lower right). ASC, apoptosis-associated speck-like protein containing a CARD; CARD, caspase-recruitment domain; DD, death domain; PYD, pyrin domain. 
the joints, characteristic of gouty inflammation (7). Although the results discussed here are essentially based on a mouse model of MSU crystal-induced inflammation, they suggest a general model of the mechanism of gouty inflammation that raises a host of new questions. For instance, it is not clear what cell types respond to IL- $1 \beta$ in the joints. We might anticipate that synoviocytes or endothelial cells play a central role in recruiting neutrophils $(13,14)$, but other cell types may be affected. For example, IL-1 $\beta$ may promote osteoclast activation and differentiation and trigger inflammatory pain hypersensitivity by acting on neurons (15).

Another important issue that remains open is the mechanism controlling the resolution phase in gout. MSU crystalinduced inflammation is generally selflimiting, suggesting that negative feedback loops control the immune response to MSU crystals. Whether such mechanisms involve an inhibition of the maturation of IL- $1 \beta$ or a desensitization to IL- $1 \beta$ responses remains to be investigated. Finally, the study of the physiological function of IL-1 $\beta$ in human gouty inflammation will undoubtedly provide new therapeutic tools to better manage the acute inflam- mation episodes in patients with gout and provide new insights into the pathology of other autoinflammatory diseases linked to deregulated IL- $1 \beta$ production.

\section{Acknowledgments}

We thank Wendy Garrett for discussions and critical reading of the manuscript.

Address correspondence to: Laurie H. Glimcher, 651 Huntington Avenue, Building FXB Room 205, Boston, Massachusetts 02115, USA. Phone: (617) 432-4846; Fax: (617) 4320084; E-mail: lglimche@hsph.harvard.edu.

1. Nuki, G., and Simkin, P.A. 2006. A concise history of gout and hyperuricemia and their treatment. Arthritis Res. Ther. 8(Suppl. 1):S1.

2. Dalbeth, N., and Haskard, D.O. 2005. Mechanisms of inflammation in gout. Rheumatology (Oxford). 44:1090-1096.

3. Faires, J.S., and McCarty, D.J. 1962. Acute arthritis in man and dog after intrasynovial infection of sodium urate crystals. Lancet. 280:682-685.

4. Shi, Y., Evans, J.E., and Rock, K.L. 2003. Molecular identification of a danger signal that alerts the immune system to dying cells. Nature. 425:516-521.

5. Heath, W.R., and Carbone, F.R. 2003. Immunology: dangerous liaisons. Nature. 425:460-461.

6. Rock, K.L., Hearn, A., Chen, C.J., and Shi, Y. 2005. Natural endogenous adjuvants. Springer Semin. Immunopathol. 26:231-246.

7. Chen, C.-J., et al. 2006. MyD88-dependent IL-1 receptor signaling is essential for gouty inflammation stimulated by monosodium urate crystals. J. Clin. Invest. 116:2262-2271. doi:10.1172/JCI28075.

8. Schnare, M., et al. 2001. Toll-like receptors control activation of adaptive immune responses. Nat. Immunol. 2:947-950.

9. O’Neill, L.A. 2006. How Toll-like receptors signal: what we know and what we don't know. Curr. Opin. Immunol. 18:3-9.

10. Martinon, F., and Tschopp, J. 2005. NLRs join TLRs as innate sensors of pathogens. Trends Immunol. 26:447-454.

11. Martinon, F., Petrilli, V., Mayor, A., Tardivel, A., and Tschopp, J. 2006. Gout-associated uric acid crystals activate the NALP3 inflammasome. Nature. 440:237-241.

12. Liu-Bryan, R., Scott, P., Sydlaske, A., Rose, D.M., and Terkeltaub, R. 2005. Innate immunity conferred by Toll-like receptors 2 and 4 and myeloid differentiation factor 88 expression is pivotal to monosodium urate monohydrate crystal-induced inflammation. Arthritis Rheum. 52:2936-2946.

13. Chapman, P.T., et al. 1997. Endothelial activation in monosodium urate monohydrate crystalinduced inflammation: in vitro and in vivo studies on the roles of tumor necrosis factor alpha and interleukin-1. Arthritis Rheum. 40:955-965.

14. Terkeltaub, R., Baird, S., Sears, P., Santiago, R., and Boisvert, W. 1998. The murine homolog of the interleukin-8 receptor CXCR-2 is essential for the occurrence of neutrophilic inflammation in the air pouch model of acute urate crystal-induced gouty synovitis. Arthritis Rheum. 41:900-909.

15. Samad, T.A., et al. 2001. Interleukin-1beta-mediated induction of Cox-2 in the CNS contributes to inflammatory pain hypersensitivity. Nature. 410:471-475

\title{
What's in a name? eNOS and anaphylactic shock
}

\section{Charles J. Lowenstein ${ }^{1}$ and Thomas Michel ${ }^{2}$}

\begin{abstract}
1Department of Medicine, Johns Hopkins University School of Medicine, Baltimore, Maryland, USA. ${ }^{2}$ Cardiovascular Division, Department of Medicine, Brigham and Women's Hospital, Harvard Medical School, Boston, Massachusetts, USA, and Veterans Affairs Boston Healthcare System,
\end{abstract} West Roxbury, Massachusetts, USA.

\begin{abstract}
In this issue of the JCI, a study by Cauwels and colleagues suggests a central role for eNOS, the endothelial isoform of nitric oxide synthase, as a mediator of anaphylaxis (see the related article beginning on page 2244). Why is an enzyme originally described as a physiological mediator of vascular homeostasis implicated in the spectacular vascular collapse that is characteristic of anaphylaxis? And is the eNOS involved in anaphylaxis necessarily exerting its effect solely in the vascular endothelium, or might this "endothelial enzyme" actually be playing a more fundamental role in an entirely different tissue? After all, what's in a name?
\end{abstract}

Mammalian NOS isoforms were originally named after the tissues in which they were first characterized and cloned. Neuronal NOS (nNOS) was used to des-

Nonstandard abbreviations used: nNOS, neuronal NOS; PAF, platelet-activating factor.

Conflict of interest: The authors have declared that no conflict of interest exists.

Citation for this article: J. Clin. Invest. 116:2075-2078 (2006). doi:10.1172/JCI29406. ignate the NOS isoform first purified and cloned from neurons, and eNOS was first isolated and characterized in endothelial cells. The NOS isoform found to be expressed in a variety of immunomodulatory cells after their stimulation with inflammatory cytokines was dubbed the inducible (or inflammation-related) NOS, or iNOS. The genes that encode nNOS, iNOS, and eNOS were officially named after the order of their discovery and characterization: NOS1, NOS2, and NOS3, respectively. However, the appellations of nNOS, iNOS, and eNOS proteins persist in the literature, perhaps for historical reasons, but these terms belie a much broader tissue distribution of these key regulatory proteins (Figure 1). Researchers discovered nNOS in nonneuronal tissues, including skeletal muscle and cardiac muscle (1). The so-called inducible iNOS now appears to be constitutively expressed in some epithelial cells in the lung and in the gastrointestinal tract as well as in myriad other cell types following their immunoactivation (2). Over the years since the original identification of eNOS in endothelial cells $(3,4)$, this "endothelial" isoform has been characterized in diverse cell types, including cardiac 